\title{
Genetic Diversity and Drug Susceptibility Profiles of Multidrug-Resistant Tuberculosis Strains in Southeast China
}

\author{
Shufang Lin' \\ Shuzhen Wei ${ }^{1}$ \\ Yong Zhao' \\ Zhisong Dai' \\ Jian Lin' \\ Yu Pang $\mathbb{( D}^{2}$
}

'Fujian Provincial Key Laboratory of Zoonosis Research, Fujian Center for Disease Control and Prevention, Fuzhou, People's Republic of China; ${ }^{2}$ Department of Bacteriology and Immunology, Beijing Key Laboratory for Drug-Resistant Tuberculosis Research, Beijing Chest Hospital, Capital Medical University, Beijing Tuberculosis and Thoracic Tumor Institute, Beijing, People's Republic of China
Correspondence: Shuzhen Wei

Fujian Center for Disease Control and Prevention, Fuzhou, People's Republic of China

Tel/Fax +8659I8343 I464

Email 499277975@qq.com

Yu Pang

Beijing Chest Hospital, Capital Medical University, Beijing, People's Republic of China

Tel/Fax +861089509359

Email pangyupound@163.com
Background: Multidrug-resistant tuberculosis (MDR-TB) isolates collected from Fujian province, China were assessed for molecular epidemiological characteristics. Analysis of isolate genotype profiles revealed that the Beijing genotype was associated with especially high drug resistance and community transmission rates.

Methods: A total of 119 MDR-TB isolates obtained from TB patients in Fujian province were typed using 24-locus mycobacterium interspersed repetitive unit-variable number tandem repeat (MIRU-VNTR) typing and spoligotyping. Drug susceptibility testing of all isolates was conducted using the L-J proportion method, with pyrazinamide (PZA) susceptibility testing conducted using the Mycobacterium Growth Indicator Tube System 960 (MGIT 960).

Results: We obtained 26 spoligotypes for the 119 isolates examined in this work. Spoligotyping results revealed that $80(67.2 \%)$ isolates possessed the Beijing family genotypic profiles. Patients aged $25-44$ years and $\geq 45$ years were most likely to be infected by non-Beijing genotypes. The percentage of clustered cases with both PZA and ofloxacin (OFLX) resistance was significantly greater than the corresponding percentage for nonclustered cases. Of 44 PZA-resistant isolates, 28 isolates (63.6\%) harbored pncA mutations, while pncA mutations were only detected in 7 (9.3\%) PZA-susceptible isolates.

Conclusion: Our data demonstrate that the Beijing genotype is the dominant lineage among MDR-TB strains circulating in Fujian. Thus, MDR-TB infections occurring within this province are not likely associated with recent transmission events. PZA and fluoroquinolone resistance profiles were found to be associated with clustered isolates. Mutation of $p n c A$ is the main driver of MDR-TB PZA resistance and is associated with mutation sites scattered throughout the entire $p n c A$ protein-coding region.

Keywords: multidrug resistance, Mycobacterium tuberculosis, genotype, pyrazinamide, pncA

\section{Introduction}

Multidrug-resistant TB (MDR-TB) infections, which result from transmission of Mycobacterium tuberculosis (MTB) strains with resistance to both rifampicin (RIF) and isoniazid (INH), continue to occur and are a major global public health issue. In 2019 an estimated 362,700 MDR-TB cases were detected worldwide, of which only $56.8 \%$ were properly diagnosed. Consequently, poor detection rates of MDR-TB infections have supported persistent community transmission of this severe form of TB. Therefore, a more comprehensive understanding of MDR-TB transmission patterns, especially within high-risk populations, is essential for the formulation of effective control strategies. 
Molecular typing is an important tool for detecting outbreaks of infectious diseases within the community while also revealing molecular characteristics of Mycobacterium tuberculosis (MTB) strains circulating in different regions. Multiple genotyping methods are often used to discriminate among strains belonging to various MTB lineages and include restriction fragment length polymorphism (RFLP) analysis, mycobacterium interspersed repetitive unit-variable-number tandem repeat (MIRU-VNTR) typing, spoligotyping, and whole-genome sequencing (WGS). Two of these methods incorporate polymerase chain reaction (PCR)-based techniques and thus are widely utilized in many geographical areas, especially in resource-limited settings. Notably, several previous studies have identified MTB lineage-specific associations between drug resistance and community transmission rates, albeit contradictory results have been reported. Therefore, there is an urgent need to elucidate correlations between genotypically defined MTB lineages and phenotypic characteristics.

Fujian province, which is located in the southeast region of China near the island of Taiwan, has a TB prevalence rate of $427.4 / 100,000$. Notably, Fujian is located at the beginning of the Maritime Silk Road, and thus its inhabitants have maintained close trade relations with Southeast Asian countries for centuries. Maintaining TB control in Fujian is challenging, due to numerous economic and trade exchanges that occur there as well as ongoing large-scale population flow into and out of the province. In order to better understand MDR-TB transmission in Fujian, we performed 24-locus MIRU-VNTR and spoligotyping to investigate molecular epidemiological characteristics of MDR-TB isolates obtained from cases in this province. In addition, we determined whether the Beijing genotype was associated with MDR-TB drug resistance and transmission in the community.

\section{Methods}

\section{Mycobacterial Isolates}

Drug surveillance was conducted in 11 randomly selected counties of the total 89 counties within Fujian province between August 2016 and August 2017 using a World Health Organization (WHO)-endorsed sampling method. All smear-positive patients who completed written informed consent forms were recruited throughout the study period for this surveillance study. Each enrolled patient was interviewed by a survey administrator using a questionnaire to collect information pertaining to patient demographic and clinical characteristics. Three sputum specimens (spot, night, and morning) were collected from each subject were used for smear microscopy and the two of which were used to generate mycobacterial cultures. All sputum smear microscopy and culturing tasks were performed by county-level laboratories. Briefly, each sputum specimen was digested with an equal volume of $4 \% \mathrm{NaOH}$ for 15 min then $0.1 \mathrm{~mL}$ of the decontaminated specimen was inoculated onto acidic Löwenstein-Jensen (L-J) medium. For eight weeks, cultures were monitored weekly for mycobacterial growth before judging cultures as negative. Culture-positive samples were sent to the Fujian provincial TB reference laboratory for bacterial species identification and drug susceptibility testing (DST).

\section{Drug Susceptibility Testing and Species Identification}

Drug susceptibility testing (DST) of positive cultures was performed using the WHO-endorsed proportional method with L-J medium. Concentrations of drugs used for testing were as follows: $0.2 \mu \mathrm{g} / \mathrm{mL}$ for isoniazid (INH), $40 \mu \mathrm{g} / \mathrm{mL}$ for rifampin (RIF), $4 \mu \mathrm{g} / \mathrm{mL}$ for streptomycin (SM), $2 \mu \mathrm{g}$ / $\mathrm{mL}$ for ethambutol (EMB), $30 \mu \mathrm{g} / \mathrm{mL}$ for kanamycin (KM), and $4 \mu \mathrm{g} / \mathrm{mL}$ for ofloxacin (OFLX). In addition, Mycobacterium species identification was conducted based on growth on medium containing $500 \mathrm{mg} / \mathrm{mL}$ of p-nitrobenzoic acid (PNB) and $5 \mathrm{mg} / \mathrm{mL}$ of 2-thiophenecarboxylic acid hydrazide (TCH). Due to the fact that in vitro DST of PZA on solid medium is of limited value, MGIT 960 was conducted using the manufacturer's protocol to determine PZA susceptibility of MTB isolates. The H37Rv MTB strain was tested with each batch of tests maintain quality control. For the proportional method, a given strain was declared resistant to a specific drug when its growth rate was at least $1 \%$ greater than the growth rate of the control. For isolates with PZA susceptibility (as determined using MGIT 960), results were automatically reported. MDR-TB was defined as TB that is resistant to both isoniazid and rifampin regardless of resistance to other antibiotics. Although the WHO has revised its definition of extensively drug-resistant tuberculosis (XDR-TB), the previous definition of XDR-TB (as MDRTB with additional resistance to OFLX and KM) was used in this study, since drug resistance surveillance for this study began in 2016 . 


\section{DNA Extraction}

Mycobacterial genomic DNA was extracted from 3-weekold mycobacterial colonies grown on L-J medium. For each isolate, one loopful of colonies was suspended in $200 \mu \mathrm{L} \mathrm{TE}$ buffer (10 mM Tris-HCH, $1 \mathrm{mM}$ EDTA) then the mycobacterial suspension was incubated at $100{ }^{\circ} \mathrm{C}$ for $30 \mathrm{~min}$ followed by centrifugation at $12,000 \times \mathrm{g}$ for $10 \mathrm{~min}$. The resulting mycobacterial DNA-containing supernatant was collected and stored at $-20{ }^{\circ} \mathrm{C}$ for later genotypic testing.

\section{Genotyping}

Spoligotyping was performed as previously described by Kamerbeek et al. ${ }^{1}$ Briefly, the mycobacterial genomic direct repeat (DR) region was amplified via PCR using primers $\mathrm{DRa}$ (biotin-labeled) and DRb. Resulting PCR products were hybridized to a set of 43 oligonucleotide probes that were covalently affixed to the surface of a membrane. ${ }^{1}$ Each spoligotype pattern result, which was based on binding of biotin-labeled PCR products to the 43 membraneimmobilized oligonucleotide probes, was expressed in binary format and compared with the spoligotypes within the SpolDB4.0 database (http://www.pasteur-guadeloupe. fr:8081/SITVIT2/) to obtain a spoligotype shared international types (SITs) code for each mycobacterial isolate.

To discriminate among MDR-TB isolates obtained in this work, the classical 24-locus MIRU-VNTR typing method was used. This test is based on PCR amplification of 24 loci, as reported previously, ${ }^{2,3}$ with H37RV and deionized water used as positive and negative controls, respectively. PCR products were electrophoretically sized using $2 \%$ agarose gels that each included a lane loaded with molecular size markers (100-bp ladder). For each isolate, the copy number of repeats at each locus was calculated and entered into an Excel spreadsheet. The discriminatory power of each locus of the 24-locus set was assessed using the Hunter-Gaston discriminatory index (HGDI). ${ }^{4}$

\section{Data Analysis}

All collected data and digital genotyping results were entered into a database using Epi Data 3.02 software (EpiData Association, Odense, Denmark). Two operators entered data separately to ensure data-entry accuracy. Genotyping results of MIRU-VNTR and spoligotyping were analyzed using Bionumerics version 5.0 (Applied Maths, Sint-Martens-Latem, Belgium). Cluster patterns were constructed using the Dice coefficient and the unweighted pair group method with arithmetic averages (UPGMA), with analysis conducted using the Bionumerics software package. Analysis using the Chi-square test was conducted to compare drug resistance rates of MTB isolates with genotypes belonging to Beijing and non-Beijing genotype families. Distributions of clustered isolates stratified according to various characteristics were also analyzed using the Chi-square test. SPSS 17.0 software (SPSS Inc, Chicago, USA) was used for all statistical analyses. Differences between comparison groups were declared significant, if corresponding P-values were $<0.05$.

\section{Results}

\section{Spoligotypes and Genotype Family Assignments}

We obtained 26 spoligotypes from the total 119 MDR-TB isolates typed in this study, with profiles corresponding to each spoligotype listed in Table 1. Based on comparative analysis of spoligotypes obtained here against the SpolDB4.0 spoligotype database, 18 matching spoligotypes were identified. The remaining eight non-matching spoligotypes were labeled "new". ST1 was the most predominant spoligotype and was detected in $63.0 \%$ of MDR-TB isolates, followed by ST50 (7, 5.9\%) and ST53 (7, 5.9\%).

Genotype family assignment analysis revealed that genotypes of $80(67.2 \%)$ of the 119 isolates belonged to the Beijing family. Of genotypes of the remaining 39 isolates, a total of $9(7.6 \%)$ genotypes were assigned to the $\mathrm{H} 3$ family, $8(6.7 \%)$ to the $\mathrm{T} 1$ family, $2(1.7 \%)$ to the $\mathrm{H}$ family, 4 (3.4\%) to the T3 family, $3(2.5 \%)$ to the H3-T3 family, $2(1.7 \%)$ to the $\mathrm{U}$ family, $1(0.8 \%)$ to the $\mathrm{T} 2$ family, $1(0.8 \%)$ to the T5 family, $1(0.8 \%)$ to the Manu2 family, and 8 are undesignated.

\section{Correlation Between Beijing Genotypes and Drug Resistance}

The highest drug resistance rate among MDR-TB isolates corresponded to resistance to EMB (62.2\%), followed by rates for SM (58.0\%), PZA (37.0\%), OFLX (24.1\%), and KM (10.9\%) (Table 2). Drug resistance rates did not differ significantly between groups of isolates with the Beijing and non-Beijing genotypes. In addition, analysis of associations between demographic characteristics and infections by MDR-TB of various genotypes (Table 3) revealed that percentages of cases infected with MTB with the Beijing family genotypes differed according to age group. As compared to patients in the $<25$ years age 
Table I Spoligotypes Shared by MDR-TB Clinical Isolates Evaluated in This Study

\begin{tabular}{|c|c|c|c|c|}
\hline $\mathbf{S I T} \mathbf{T}^{\mathbf{a}}$ & Lineage/Sublineage $^{b}$ & Spoligotypes in Binary & Octal & Noc.(\%) \\
\hline 1 & Beijing & 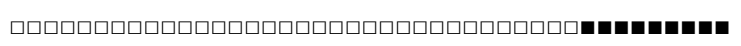 & 000000000003771 & 75 \\
\hline 250 & Beijing & 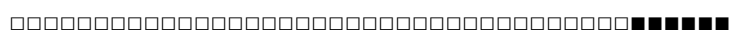 & 000000000000371 & 3 \\
\hline 190 & Beijing-like & 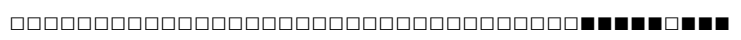 & 000000000003731 & I \\
\hline 585 & Beijing-like & 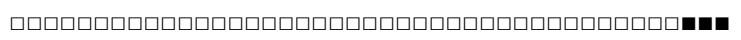 & 000000000000031 & I \\
\hline 36 & H3-T3 & 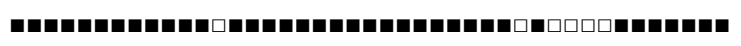 & $777,737,777,720,77 \mid$ & 3 \\
\hline 50 & H3 & 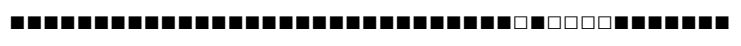 & $777,777,777,720,771$ & 7 \\
\hline 467 & $\mathrm{H} 3$ & 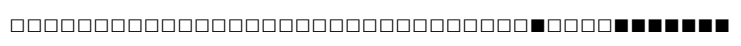 & 000000000020771 & 2 \\
\hline 742 & $\mathrm{H}$ & 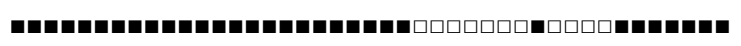 & $777,777,770,020,771$ & I \\
\hline 946 & $\mathrm{H}$ & ' & $777,777,740,020,771$ & I \\
\hline 53 & TI & 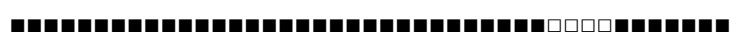 & $777,777,777,760,77 \mid$ & 7 \\
\hline 334 & $\mathrm{TI}$ & 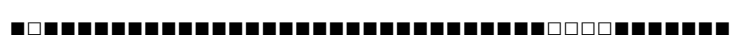 & $577,777,777,760,771$ & I \\
\hline 1098 & $\mathrm{~T} 2$ & 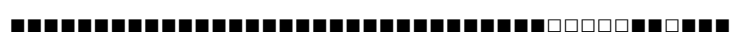 & $777,777,777,760,331$ & I \\
\hline 254 & T5-RUSI & 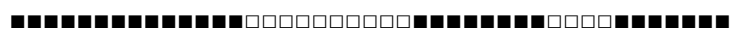 & $777,760,007,760,771$ & 1 \\
\hline 1163 & T3 & 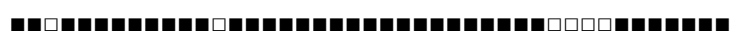 & $677,737,777,760,771$ & I \\
\hline 37 & T3 & 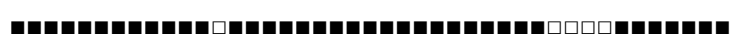 & $777,737,777,760,771$ & 3 \\
\hline 1462 & $U$ & 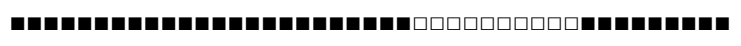 & $777,777,770,003,771$ & 1 \\
\hline 246 & $u$ & 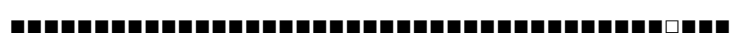 & $777,777,777,777,731$ & I \\
\hline \multirow[t]{9}{*}{1634} & MANU2 & 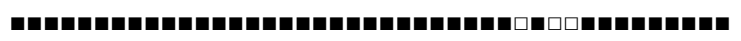 & $777,777,777,723,771$ & I \\
\hline & Undesignated & 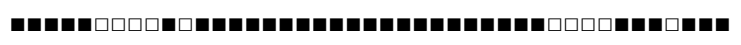 & $760,577,777,760,731$ & I \\
\hline & Undesignated & 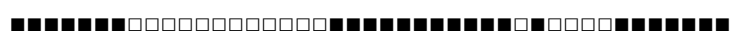 & $774,000,377,720,771$ & I \\
\hline & Undesignated & 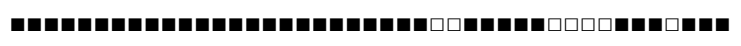 & $777,777,774,760,731$ & I \\
\hline & Undesignated & 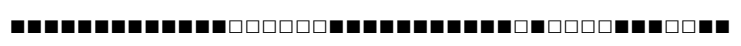 & $777,740,377,720,7 \mid I$ & I \\
\hline & Undesignated & " & $777,777,770,000,031$ & I \\
\hline & Undesignated & 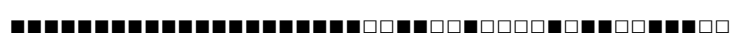 & $777,777,7|4,4| 3,160$ & I \\
\hline & Undesignated & 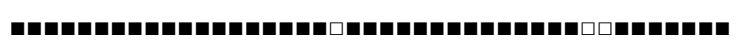 & $777,777,577,774,77 \mid$ & I \\
\hline & Undesignated & 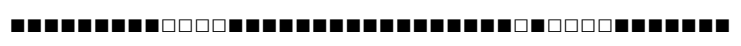 & $777,037,777,720,77 \mid$ & I \\
\hline
\end{tabular}

Notes: ${ }^{a}$ SIT from SpoIDB4.0, ${ }^{b}$ Representing spoligotypes families as assigned in the SpolDB4.0 database, ${ }^{C}$ Number of strains with an identical SIT. $\square$ lt means there is no hybrid signal; $\mathbf{a}$ t means there is a hybrid signal.

group (the control), patients in $25-44$ years and $\geq 25$ years age groups were more likely to be infected with MTB with non-Beijing family genotypes. By contrast, infection rates according to gender, education level, occupation, residence, or treatment history did not significantly differ between Beijing and non-Beijing genotypes.

Table 2 The Drug Resistant Rates of Different Families Compared in This Study

\begin{tabular}{|l|l|l|l|l|}
\hline Resistant & $\begin{array}{l}\text { Beijing } \\
(\mathbf{N}=80, \%)\end{array}$ & $\begin{array}{l}\text { Non-Beijing } \\
(\mathbf{N}=39, \%)\end{array}$ & $\begin{array}{l}\text { Total } \\
\mathbf{( N = 1 ~ I 9 , ~} \\
\text { \%) }\end{array}$ & P value \\
\hline EMB & $49(61.3)$ & $25(64.1)$ & $74(62.2)$ & 0.763 \\
SM & $49(61.3)$ & $20(51.3)$ & $69(58.0)$ & 0.301 \\
PZA & $31(38.8)$ & $13(33.3)$ & $44(37.0)$ & 0.566 \\
OFLX & $24(30.0)$ & $8(20.5)$ & $32(26.9)$ & 0.273 \\
KM & $9(I 1.3)$ & $4(10.3)$ & $13(10.9)$ & 0.870 \\
\hline
\end{tabular}

Abbreviations: EMB, ethambutol; SM, streptomycin; PZA, pyrazinamide; OFLX, ofloxacin; KM, kanamycin; $\mathrm{N}$, numbers of the strains.
Of the 119 MDR-TB cases studied here, $76.5 \%$ were male and $23.5 \%$ were female. Most MDR-TB cases were concentrated within the $>45$ and 25-44 age groups, accounting for $54.6 \%$ and $37.8 \%$ of cases, respectively. With regard to the $<25$ years age group, for cases belonging to $25-44$ and $>45$ years age groups, infection rates of MTB with non-Beijing family genotypes were greater than corresponding infection rates associated with Beijing family genotypes. No statistically significant differences of case demographic characteristics (eg, gender, age, education, farming occupation, coastal area residence, treatment history) or PZA resistance were associated with MDR-TB genotype (Beijing versus non-Beijing).

\section{4-Locus MIRU-VNTR Genotyping}

As shown in Figure 1, genes of MDR-TB isolates exhibited high numbers of polymorphisms, as demonstrated based on 24-locus MIRU-VNTR genotyping results. Typing results revealed that for the 119 isolates analyzed in this study, 112 distinct genotypes were detected, of 
Table 3 Characteristics in MDR-TB Strains Between the Beijing and Non-Beijing Family

\begin{tabular}{|c|c|c|c|c|c|}
\hline \multirow[t]{2}{*}{ Characteristics } & \multicolumn{3}{|c|}{ Spoligotypes } & \multirow[t]{2}{*}{$P$ value } & \multirow[t]{2}{*}{ OR $(95 \% \mathrm{Cl})$} \\
\hline & Beijing ( $\mathbf{N}=\mathbf{8 0}, \%)$ & Non-Beijing ( $N=39, \%)$ & Total $(\mathbf{N}=1 \mid 9, \%)$ & & \\
\hline \multicolumn{6}{|l|}{ Gender } \\
\hline Male & $60(75.0)$ & $31(79.5)$ & $91(76.5)$ & 0.59 & $0.77(0.31-1.96)$ \\
\hline Female & $20(25.0)$ & $8(20.5)$ & $28(23.5)$ & - & I.0(Ref.) \\
\hline \multicolumn{6}{|l|}{ Age group, years } \\
\hline $0-24$ & $9(11.3)$ & $0(0)$ & $9(7.6)$ & - & I.0(Ref.) \\
\hline $25-44$ & $28(35.0)$ & $17(43.6)$ & $45(37.8)$ & 0.03 & $1.61(1.28-2.02)$ \\
\hline$\geq 45$ & $43(53.8)$ & $22(56.4)$ & $65(54.6)$ & 0.04 & $1.51(1.27-1.80)$ \\
\hline \multicolumn{6}{|l|}{ Farmer } \\
\hline Yes & $43(53.8)$ & $23(59.0)$ & $66(55.5)$ & - & I.0(Ref.) \\
\hline No & $37(46.3)$ & $16(41.0)$ & $53(44.5)$ & 0.59 & $0.8 I(0.37-1.75)$ \\
\hline \multicolumn{6}{|l|}{ Education } \\
\hline Illiterate & $16(20.0)$ & $9(23.1)$ & $25(21.0)$ & - & I.0(Ref.) \\
\hline Literate & $64(80.0)$ & $30(76.9)$ & $94(79.0)$ & 0.70 & $0.83(0.33-2.10)$ \\
\hline \multicolumn{6}{|l|}{ Areas } \\
\hline Coastal areas & $61(76.3)$ & $35(89.8)$ & $80(67.2)$ & - & I.0(Ref.) \\
\hline Mainland areas & $19(23.8)$ & $4(10.3)$ & $39(32.8)$ & 0.08 & $0.37(0.12-1.67)$ \\
\hline \multicolumn{6}{|l|}{ Treatment history } \\
\hline New case & $4 I(5 \mid .3)$ & $18(46.2)$ & $59(49.6)$ & - & I.0(Ref.) \\
\hline Retreated & $39(48.8)$ & $21(53.9)$ & $60(50.4)$ & 0.60 & $1.23(0.57-2.64)$ \\
\hline
\end{tabular}

Abbreviation: $\mathrm{N}$, numbers of the strains.

which 105 possessed unique repeat patterns and 7 belonged to genotypic clusters. Each cluster included two isolates, for a clustering rate of $11.8 \%$ (14/119). The discriminatory power of each locus is summarized in Table 4. The highest HGDI value was associated with locus QUB11b (HGDI=0.796), whereas MIRU02 and MIRU20 loci exhibited no sublineage-level discriminatory ability and thus were not useful for MDR-TB isolate classification $(\mathrm{HGDI}=0)$. In addition, HGDI values obtained for 4 loci (ETRE, MTUB21, QUB11b, and QUB26) were greater than 0.6 and thus were considered highly discriminatory loci.

\section{Demographic and Clinical Characteristics of Clustered MDR-TB Isolates}

We further compared socio-demographic case characteristics and drug-resistance profiles of MDR-TB isolates between clustered and non-clustered case groups. As shown in Table 5, cluster-associated percentages of isolates exhibiting PZA resistance and OFLX resistance were significantly higher than corresponding percentages associated with non-clustered cases. Conversely, percentages of resistant isolates associated with other drug susceptibility profiles did not differ significantly between clustered and non-clustered cases. Furthermore, data analysis revealed no statistically significant differences in several demographic characteristics, including gender, age, and treatment history between clustered and non-clustered cases.

\section{Mutations of the pncA Gene}

Correlation analysis to identify associations between PZA resistance and resistance to other anti-TB drugs revealed notably high SM and EMB resistance rates in PZAresistant isolates $(70.4 \%$ and $81.8 \%$, respectively). These rates were significantly higher than corresponding rates for PZA-susceptible isolates, of $50.7 \%$ and $52.0 \%$, respectively ( $P=0.035$ for $\mathrm{SM}$ and $P=0.001$ for EMB).

Of 44 PZA-resistant isolates, $28(63.6 \%)$ harbored mutations within pncA, whereas only 7 (9.3\%) PZAsusceptible isolates harbored $p n c A$ mutations. As shown in Table 6, a high degree of $p n c A$ mutational diversity was observed, with 25 different mutations detected in MDRTB isolates, including 23 single-nucleotide substitutions 


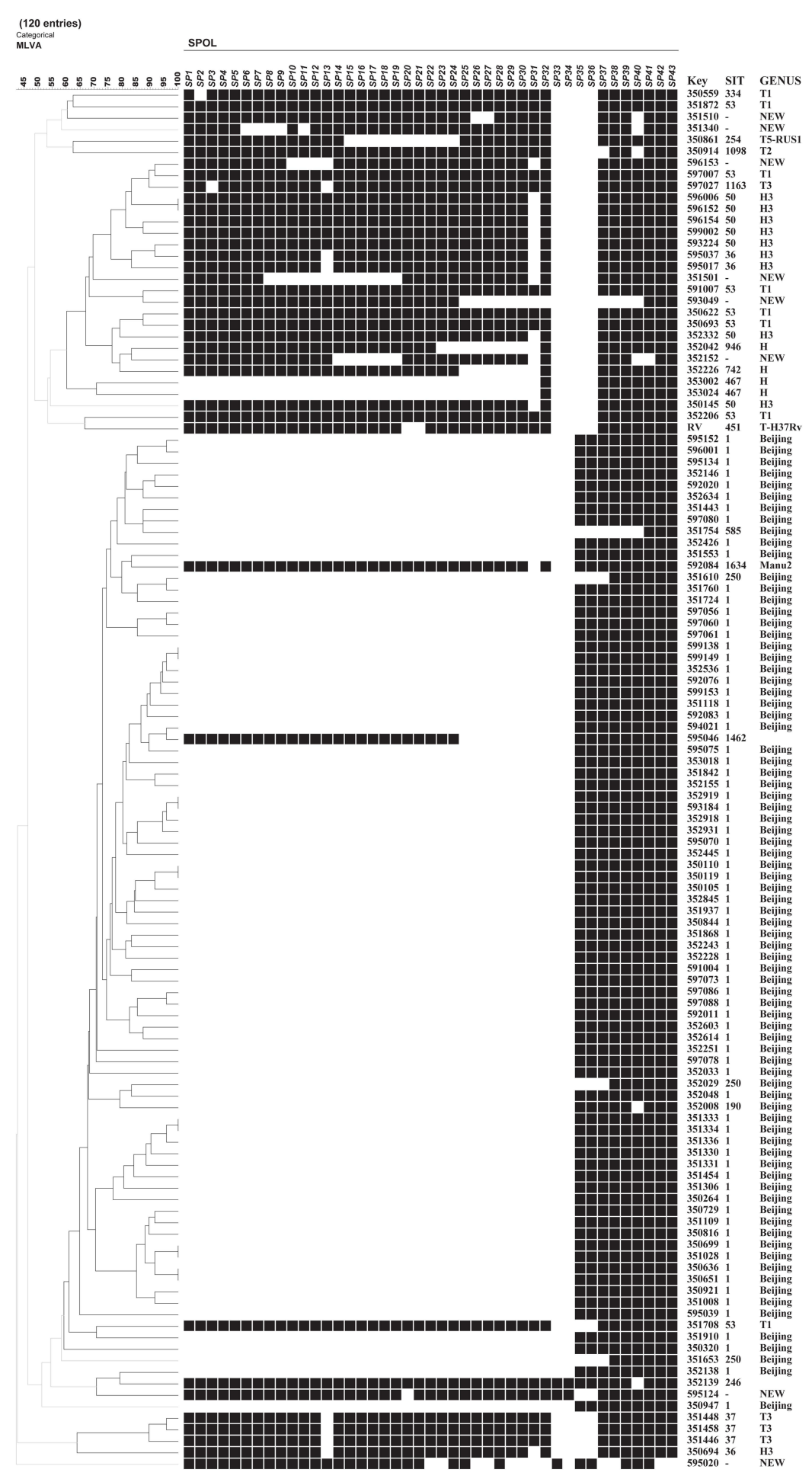

Figure I MDR-TB isolates genotyped by 24 loci MIRU-VNTR.

and 2 frameshift mutations. The most prevalent mutation associated with PZA resistance was detected in pncA codon 104, resulting in an amino acid substitution of serine (Ser) for isoleucine (Ile). The second most commonly mutated codon was codon 68 , with mutations of this codon noted in three isolates $(6.8 \%, 3 / 44)$. In addition, a synonymous mutation involving an $\mathrm{A}$ to $\mathrm{C}$ transversion at $p n c A$ position 345 was detected in two PZA-susceptible isolates, while two PZA-susceptible isolates carried nonsynonymous mutations of $p n c A$, including one mutation 
Table 4 HGDI of 24-Loci VNTR for MDR-TB Strains

\begin{tabular}{|c|c|c|c|c|c|c|c|}
\hline $\begin{array}{l}\text { 24-Loci } \\
\text { VNTR }\end{array}$ & $\begin{array}{l}\text { HGDI (All } \\
\text { Strains) }\end{array}$ & $\begin{array}{c}\text { HGDI } \\
\text { (Beijing) }\end{array}$ & $\begin{array}{l}\text { HGDI (Non- } \\
\text { Beijing) }\end{array}$ & $\begin{array}{c}\text { Size Range } \\
\text { Observed }\end{array}$ & $\begin{array}{c}\text { No. of Alleles } \\
\text { Observed }\end{array}$ & I5-LociVNTR & Diversity* \\
\hline QUBIIB & 0.7958 & 0.7282 & 0.8151 & $\mathrm{I}-7$ & 7 & $\sqrt{ }$ & High \\
\hline QUB26 & 0.7260 & 0.6788 & 0.7989 & $1-9$ & 8 & $\sqrt{ }$ & High \\
\hline MTUB2I & 0.7125 & 0.5794 & 0.6734 & $1-6$ & 6 & $\sqrt{ }$ & High \\
\hline ETRE & 0.6627 & 0.4756 & 0.6005 & $2-5$ & 4 & $\sqrt{ }$ & High \\
\hline MIRU23 & 0.5538 & 0.5377 & 0.6221 & $1-4$ & 4 & & Moderate \\
\hline MTUB04 & 0.5275 & 0.2731 & 0.5223 & $1-4$ & 6 & $\sqrt{ }$ & Moderate \\
\hline MIRU26 & 0.5177 & 0.2684 & 0.5547 & $2-6$ & 5 & $\sqrt{ }$ & Moderate \\
\hline MIRUIO & 0.5044 & 0.3209 & 0.5533 & $1-5$ & 5 & $\sqrt{ }$ & Moderate \\
\hline QUB4I56C & 0.5031 & 0.5294 & $0.456 \mathrm{I}$ & $1-4$ & 4 & $\sqrt{ }$ & Moderate \\
\hline MIRU39 & 0.4883 & 0.3364 & 0.5776 & $2-4$ & 4 & $\sqrt{ }$ & Moderate \\
\hline MIRU27 & 0.4737 & 0.3861 & 0.6262 & $1-4$ & 4 & & Moderate \\
\hline ETRA & 0.4519 & 0.1861 & 0.3645 & $2-8$ & 5 & $\sqrt{ }$ & Moderate \\
\hline MIRUI6 & 0.4243 & 0.3820 & 0.5304 & $1-4$ & 4 & $\sqrt{ }$ & Moderate \\
\hline MTUB30 & 0.4078 & 0.0497 & 0.3023 & $2-4$ & 3 & $\sqrt{ }$ & Moderate \\
\hline MIRU40 & 0.4014 & 0.3285 & 0.5304 & $1-4$ & 4 & $\sqrt{ }$ & Moderate \\
\hline ETRB & 0.3896 & 0.1415 & 0.5452 & $1-3$ & 3 & & Moderate \\
\hline MTUB29 & 0.3493 & 0.2902 & 0.4845 & $2-5$ & 4 & & Moderate \\
\hline MTUB39 & 0.3197 & 0.2544 & 0.4602 & $1-6$ & 6 & & Moderate \\
\hline ETRC & 0.2657 & 0.2326 & 0.3563 & $I-5$ & 5 & $\sqrt{ }$ & Poor \\
\hline ETRD & 0.2238 & 0.1617 & 0.3644 & $1-6$ & 6 & $\sqrt{ }$ & Poor \\
\hline MTUB34 & 0.0736 & 0.0494 & 0.1457 & $2-4$ & 3 & & Poor \\
\hline MIRU24 & 0.0083 & 0 & 0.0513 & $1-2$ & 2 & & Poor \\
\hline MIRU02 & 0 & 0 & 0 & 1 & I & & Poor \\
\hline MIRU20 & 0 & 0 & 0 & I & I & & Poor \\
\hline
\end{tabular}

Notes: HGDI represents the value of allelic diversity of each locus. ${ }^{*}$ The VNTR loci were designated as high, moderate, and poor discriminatory at $h$ of $>0.6, \geqslant 0.3$ and $\leqslant 0.6$, and $<0.3$, respectively.

detected in codon 136 and one mutation detected in codon 383 .

\section{Discussion}

This study provides novel insights into population structure-based rates and genotype diversity of MDR-TB isolates obtained from TB patients enrolled at drug resistance surveillance sites in Southeast China, as determined using 24-locus MIRU-VNTR typing and spoligotyping. The results revealed that MDR-TB with Beijing family genotypes was predominant in Fujian province, with a prevalence rate of $67.2 \%$. However, this rate was lower than corresponding rates previously reported for Beijing (92.6\%), ${ }^{5}$ Hebei $(90.5 \%),{ }^{2}$ Heilongjiang (89.5\%), ${ }^{6}$ Jiangsu $(80.4 \%){ }^{3}$ Henan $(83.5 \%)$, Zhejiang $(71.6 \%){ }^{7}$ and Yunnan $(70.5 \%)^{8}$ and greater than rates found in Guangxi $(55.3 \%)^{5}$ and Taipei (52.5\%). ${ }^{9}$ Taken together, these results demonstrate variable Beijing family-type genotype distributions among different geographic areas across China.
According to results of several previous studies, Beijing family genotypes were more highly correlated with MDR phenotypes than were non-Beijing family genotypes, ${ }^{8,10-12}$ indicating that genotype family may be strongly associated with MDR-TB transmission. By contrast, in this study, no association was found between the Beijing genotype and various other drug resistance profiles, as was also reported previously. ${ }^{13}$ Despite the lack of an association between the Beijing genotype and nonMDR drug resistance phenotypes in this work, we did observe that SM and EMB drug resistance rates of PZAresistant MDR isolates were higher than corresponding rates for PZA-susceptible MDR isolates. This result should be taken into account when developing treatment plans for patients infected with drug-resistant MTB.

Besides Beijing family isolates, non-Beijing family isolates were also characterized in this study. These isolates possessed H, H3, H3-T3, T1, T2, T3, T5-RUS1, U, MANU2 genotypes and eight new genotypes, indicating appreciable diversity of non-Beijing family genotypes in 
Table 5 Characteristics in MDR-TB Strains Between the Clustered and Individual Strain Family

\begin{tabular}{|c|c|c|c|c|c|}
\hline \multirow{2}{*}{ Characteristics } & \multicolumn{2}{|c|}{ Strains } & \multirow[t]{2}{*}{ Total (N= I | 9,\%) } & \multirow[t]{2}{*}{$P$ value } & \multirow[t]{2}{*}{ OR $(95 \% \mathrm{Cl})$} \\
\hline & Clustered $(\mathrm{N}=\mid 4, \%)$ & Non-Clustered ( $N=105, \%)$ & & & \\
\hline \multicolumn{6}{|l|}{ Gender } \\
\hline Male & $\mathrm{II}(78.6)$ & $80(76.2)$ & $91(76.5)$ & 0.84 & $0.87(0.23-3.38)$ \\
\hline Female & $3(21.4)$ & $25(23.8)$ & $28(23.5)$ & - & I.0(Ref.) \\
\hline \multicolumn{6}{|l|}{ Age group, years } \\
\hline $0-24$ & $\mathrm{I}(7 . \mathrm{I})$ & $8(7.6)$ & $9(7.6)$ & - & I.0(Ref.) \\
\hline $25-44$ & $4(28.6)$ & $4 I(39.1)$ & $45(37.8)$ & 0.84 & $1.28(0.13-13.02)$ \\
\hline$\geq 45$ & $9(64.3)$ & $56(53.3)$ & $65(54.6)$ & 0.82 & $0.78(0.09-6.98)$ \\
\hline \multicolumn{6}{|l|}{ Family } \\
\hline Beijing & $12(85.7)$ & $68(64.8)$ & $80(67.2)$ & 0.12 & $0.3 \mid(0.07-I .44)$ \\
\hline NonBeijing & $2(14.3)$ & $37(35.2)$ & $39(32.8)$ & - & I.0(Ref.) \\
\hline \multicolumn{6}{|l|}{ Treatment history } \\
\hline New case & $9(64.3)$ & $50(47.6)$ & $59(49.6)$ & 0.24 & $0.5 I(0.16-1.61)$ \\
\hline Retreated & $5(35.7)$ & $55(52.4)$ & $60(50.4)$ & - & I.0(Ref.) \\
\hline \multicolumn{6}{|l|}{ PZA } \\
\hline Susceptible & $5(35.7)$ & $70(66.7)$ & $75(63.0)$ & 0.02 & $3.6(1.12-11.56)$ \\
\hline Resistant & $9(64.3)$ & $35(33.3)$ & $44(37.0)$ & - & I.0(Ref.) \\
\hline \multicolumn{6}{|l|}{ SM } \\
\hline Susceptible & $6(42.9)$ & $44((41.9)$ & $50(42.0)$ & 0.95 & $0.96(0.31-2.97)$ \\
\hline Resistant & $8(57.1)$ & $6 I(58.1)$ & $69(57.9)$ & - & I.0(Ref.) \\
\hline \multicolumn{6}{|l|}{ EMB } \\
\hline Susceptible & $4(28.6)$ & $40(38.1)$ & $44(37.0)$ & 0.49 & I.54(0.45-5.24) \\
\hline Resistant & $10(71.4)$ & $65(61.9)$ & $75(63.0)$ & - & I.0(Ref.) \\
\hline \multicolumn{6}{|l|}{ OFLX } \\
\hline Susceptible & $7(50.0)$ & $80(76.2)$ & $97(81.5)$ & 0.04 & $3.2(1.02-10.00)$ \\
\hline Resistant & $7(50.0)$ & $25(23.8)$ & $32(26.9)$ & & \\
\hline \multicolumn{6}{|l|}{ KM } \\
\hline Susceptible & $13(92.9)$ & $93(88.6)$ & 106(89.1) & 0.63 & $0.6(0.07-4.97)$ \\
\hline Resistant & $\mathrm{I}(7.1)$ & $12(1 \mid .4)$ & $13(10.9)$ & & \\
\hline
\end{tabular}

Abbreviations: PZA, pyrazinamide; SM, streptomycin; EMB, ethambutol; OFLX, ofloxacin; KM, kanamycin; N, numbers of the strains.

Fujian province. Spoligotyping of isolates with the eight new genotypes revealed that the genotypes of six of these isolates were unique and were associated with nonepidemiologically linked cases originating from different counties. Thus, results of geographic analysis of genotypic lineages of isolates obtained in this work suggest that these isolates belong to lineages that have already spread throughout Fujian province. Interestingly, the reported worldwide prevalence of isolates with the MANU2 family genotype is low, with only two isolates reported in South Africa; ${ }^{14}$ of these isolates, one was mono-INH resistant, and the other was mono-RFP resistant. By contrast, the single MANU2 family isolate detected in this study was sensitive to 11 other anti-tuberculosis drugs. Notably, the $\mathrm{T}$ and $\mathrm{H}$ family genotypes shown here to be highly prevalent in Fujian are also prevalent in Europe and South America. ${ }^{15,16}$ The underlying reason for common high prevalence of these genotypes in Fujian and geographically distant regions may reflect the fact that Fujian province is located on the southeast coast of China and its people engage in frequent economic and trade activities with peoples from other parts of the world. Moreover, accelerated development of Fujian's tourism industry has led to greater population mobility. Furthermore, the perennial hot and humid climate in Fujian province may support transmission of non-Beijing family MDR-TB. 
Table 6 The Mutations in pncA Detected in the PZA Resistance MDR Strains

\begin{tabular}{|c|c|c|c|c|}
\hline \multicolumn{2}{|c|}{$\begin{array}{l}\text { PZA } \\
\text { Susceptibility }\end{array}$} & \multirow[t]{2}{*}{ pncA Mutation } & \multirow[t]{2}{*}{ Codon } & \multirow[t]{2}{*}{ Amino Acid Change } \\
\hline $\mathbf{S}$ & $\mathbf{R}$ & & & \\
\hline 0 & 2 & $22 \mathrm{G} \rightarrow \mathrm{A}$ & GACBAAC & $\mathrm{D} 8 \mathrm{~N}$ \\
\hline 0 & I & $29 A \rightarrow C$ & CAGIOCCG & QIOP \\
\hline 0 & I & $42 \mathrm{C} \rightarrow \mathrm{A}$ & TGCI4TGA & $\mathrm{Cl}$ 4end \\
\hline 0 & I & $\mathrm{I} 37 \mathrm{C} \rightarrow \mathrm{A}$ & GCA46GAA & A46E \\
\hline 0 & I & $\mathrm{I} 37 \mathrm{C} \rightarrow \mathrm{T}$ & GCA46GTA & A46V \\
\hline 0 & I & $152 \mathrm{~A} \rightarrow \mathrm{C}$ & CAC5ICCC & $\mathrm{H} 5 \mathrm{IP}$ \\
\hline 0 & 3 & $202 T \rightarrow C$ & TGG68CGG & W68R \\
\hline 0 & I & $23 \mathrm{IIns}$ & 23 IInsert & Frameshift \\
\hline 0 & I & $246 \mathrm{~A} \rightarrow \mathrm{C}$ & САТ $82 C C T$ & $\mathrm{H} 82 \mathrm{P}$ \\
\hline 0 & 4 & $3 I I G \rightarrow T$ & AGCI04ATC & SI04I \\
\hline 0 & I & $347 \mathrm{~T} \rightarrow \mathrm{C}$ & CTGII6CCG & LII6P \\
\hline 0 & 2 & $3891 \mathrm{~ns}$ & 389 Insert & Frameshift \\
\hline 0 & I & $403 \mathrm{~A} \rightarrow \mathrm{C}$ & $\mathrm{ACCI} 35 \mathrm{CCC}$ & TI35P \\
\hline 0 & 2 & $42 \mathrm{IC} \rightarrow \mathrm{T}$ & CAGI4ITAG & QI4lend \\
\hline 0 & I & $422 \mathrm{~A} \rightarrow \mathrm{C}$ & CAGI4ICCG & QI4IP \\
\hline 0 & I & $478 \mathrm{~A} \rightarrow \mathrm{G}$ & ACAI60GCA & $\mathrm{TI} 60 \mathrm{~A}$ \\
\hline 0 & I & $490 \mathrm{~T} \rightarrow \mathrm{C}$ & TCGI64CCG & SI64P \\
\hline 0 & I & $523 \mathrm{~A} \rightarrow \mathrm{G}$ & ATGI75GTG & MI75V \\
\hline \multirow[t]{2}{*}{0} & 1 & $278 \mathrm{~T} \rightarrow \mathrm{G}, 319 \mathrm{G} \rightarrow \mathrm{A}, 376 \mathrm{G} \rightarrow \mathrm{A}$, & TCG93TCA, GAGII2AAG, CAGI4ICCG, & V93G, EII2K, QI4IP, \\
\hline & & $422 \mathrm{~A} \rightarrow \mathrm{C}, 54 \mathrm{IG} \rightarrow \mathrm{A}$ & GATI26AAT, GAGI8IAAG & DI26N, EI8IK \\
\hline 1 & 0 & $136 \mathrm{G} \rightarrow \mathrm{A}$ & GCA46ACA & A46T \\
\hline 2 & 0 & $345 \mathrm{~A} \rightarrow \mathrm{C}$ & CCAII5CCC & Synonymous mutation \\
\hline 1 & 0 & $383 \mathrm{~T} \rightarrow \mathrm{G}$ & GTCI28GGC & VI28G \\
\hline I & 0 & $396 \mathrm{~T} \rightarrow \mathrm{C}$ & GGTI32GGC & Synonymous mutation \\
\hline I & 0 & $398 \mathrm{~T} \rightarrow \mathrm{C}$ & ATTI33ACT & II $33 \mathrm{~T}$ \\
\hline 1 & I & $464 \mathrm{~T} \rightarrow \mathrm{G}$ & GTGI55GGG & VI55G \\
\hline
\end{tabular}

Abbreviations: PZA, pyrazinamide; S, susceptible; $R$, resistance.

Nevertheless, in order to investigate these possible explanations, more extensive typing of clinical samples is needed using typing methods with greater discriminatory power.

The resolution provided by 24-locus VNTR has previously been reported to be comparable to that of IS6110RFLP. ${ }^{17}$ However, VNTR resolution obtained here for Fujian MDR-TB isolates was greater than that obtained in those previous studies. Of note, all genotypic clusters detected in this study contained at most two isolates. The small cluster sizes and high discriminatory power of the VNTR assay used here indicate that the MDR-TB infections in Fujian might not be associated with recent transmission events. Since 2013, approximately $43.0 \%$ of counties in Fujian have been equipped with Xpert MTB/RIF testing labs. Utilization of this novel RIF resistance-based TB assay is important for maintaining TB control, since it may reduce MDT-TB time to diagnosis as compared to that achievable using conventional phenotypic DST, thereby supporting timely initiation of appropriate treatment and prevention of community transmission. In addition, Fujian hosts a large, highly diverse and mobile migrant population that may harbor MDR-TB strains with diverse genotypes to further explain the high level of discriminatory power of genotypic typing results obtained here. Interestingly, only PZA and FQ resistance straits were found to be associated with clustered isolates. Although the underlying reasons for this phenomenon remain unclear, we hypothesize that additional PZA and FQ resistance traits have low fitness costs while also driving MDR-TB transmission. In line with this hypothesis, we found marked diversity of pncA mutations associated with PZA resistance, suggesting that mutations within $p n c A$ have little impact on the fitness of tubercle bacilli. Nevertheless, further experimental evidence is urgently needed to test this hypothesis.

Although resolution values obtained from 24-locus VNTR typing of the 119 isolates tested here were as 
high as 0.999 , two loci (MIRU02 and MIRU20) lacked polymorphisms. Meanwhile, poor resolution values were obtained for four other loci, ETRC, ETRD, MIRU24, and MTUB34 (HGDI $<0.3$ ), thus indicating that these six loci were unsuitable for use in genotyping of MDR-TB isolates from cases in Fujian province. By contrast, high-resolution results were obtained for loci with the highest polymorphism rates, namely QUB11B, QUB26, MTUB21, and ETRE (HGDI >0.6). Generally, genetic polymorphisms of epidemic TB strains have been found to vary in different regions, with even Beijing family strains shown to exhibit different VNTR types in different regions. Although here the overall resolution obtained using 24locus VNTR of MDR-TB strains was high, some loci were not suitable for typing MDR-TB in Fujian province. However, VNTR typing using a larger set of loci would be relatively complicated and costly, prompting this study to formulate an optimal set of fewer loci to specifically monitor the MDR-TB epidemic in Fujian province.

Numerous studies reported in the literature have shown that MTB pncA mutations cause loss or reduction of pyrazinamide enzyme activity for converting PZA to POA, resulting in PZA resistance. Nevertheless, in this study, only $63.6 \%$ of PZA-resistant isolates harbored genetic mutations within $p n c A$, while in previous studies, concurrence of phenotypic PZA resistance and pncA mutations varied greatly, ranging from $41.2 \%$ in Taiwan ${ }^{18}$ to $90.5 \%$ in Shanghai. ${ }^{19}$ These differences have been attributed to variations of specific $p n c A$ gene mutation rates in different geographic regions. Additionally, $p n c A$ mutations are highly diverse and might not be detected using current diagnostic methods that only screen for mutations within known mutational hotspots. As an alternative, DNA sequencing of the entire $p n c A$ gene would likely be more effective for detecting $p n c A$ mutations associated with PZA resistance.

This study had several limitations. First, the sample size of isolates was small and was not likely representative of MTB strains circulating in all areas of China, since isolates characterized here were derived from cases visiting drug resistance surveillance sites only within Fujian, where drug resistance rates are lower than rates elsewhere in China. Thus, we would increase sample size to increase the reliability of our findings in future studies. Second, whole-genome sequencing (WGS) outperforms conventional typing methods, due to its greater discriminatory power. However, such testing was not conducted in this study, potentially leading to overestimation of clustering rates. Finally, epidemiologic associations among clusters were not analyzed, due to incomplete TB patient follow-up. Thus, future investigations are needed which incorporate patient tracking in order to identify infection factors.

In conclusion, we describe for the first time the genetic diversity of MTB isolates collected from TB patients in Fujian province, as determined using 24-locus MIRUVNTR combined with spoligotyping and DST. Our data demonstrated that the Beijing family lineage was the predominant genotypic lineage associated with MDR-TB strains in southeastern China. In addition, MDR-TB infections in Fujian were not associated with recent transmission events, while PZA and FQ resistance traits were associated with clustered isolates. Mutation of $p n c A$ is the main mechanism driving the generation of PZAresistant MDR strains, with scattered mutation sites detected throughout the entire $p n c A$ protein-coding region.

\section{Ethical Approval Statement}

This project was approved by the Ethical Committee of the Fujian Province Center for Disease Control and Prevention (CDC). The written informed consent forms were obtained from the enrolled patients prior to study enrolment. Ethics have been respected in the whole period. The study was conducted in accordance with the Declaration of Helsinki.

\section{Acknowledgments}

We thank all technical staff in the municipal and county level TB laboratory for their contribution in collecting strains. And we also appreciate to the help from the staff in Chinese National TB Reference Laboratory.

\section{Funding}

This study was sponsored by Fujian provincial health technology project (2019-ZQN-28), the Construction of Fujian Provincial Scientific and Technological Innovation Platform (2019Y2001), the Beijing Hospitals Authority Ascent Plan (DFL20191601), and the Beijing Hospitals Authority Clinical Medicine Development of Special Funding (ZYLX202122). The funder had no role in study design, data collection and analysis, decision to publish, or preparation of the manuscript.

\section{Disclosure}

The authors declare that they have no conflicts of interest. 


\section{References}

1. Kamerbeek J, Schouls L, Kolk A, et al. Simultaneous detection and strain differentiation of Mycobacterium tuberculosis for diagnosis and epidemiology. $J$ Clin Microbiol. 1997;35(4):907-914. doi:10.1128/jcm.35.4.907-914.1997

2. Li Y, Cao X, Li S, et al. Characterization of Mycobacterium tuberculosis isolates from Hebei, China: genotypes and drug susceptibility phenotypes. BMC Infect Dis. 2016;16(1):107. doi:10.1186/s12879016-1441-2

3. Liu Q, Yang D, Xu W, et al. Molecular typing of Mycobacterium tuberculosis isolates circulating in Jiangsu province, China. BMC Infect Dis. 2011;11(1):288. doi:10.1186/1471-2334-11-288

4. Hunter PR. Reproducibility and indices of discriminatory power of microbial typing methods. J Clin Microbiol. 1990;28(9):1903-1905. doi:10.1128/jcm.28.9.1903-1905.1990

5. Dong H, Liu Z, Lv B, et al. Spoligotypes of Mycobacterium tuberculosis from different provinces of China. J Clin Microbiol. 2010;48 (11):4102-4106. doi:10.1128/JCM.00549-10

6. Wang J, Liu Y, Zhang CL, et al. Genotypes and characteristics of clustering and drug susceptibility of Mycobacterium tuberculosis isolates collected in Heilongjiang province, China. $J$ Clin Microbiol. 2011;49(4):1354-1362. doi:10.1128/JCM.02274-10

7. Liu Z, Pang Y, Chen S, et al. A first insight into the genetic diversity and drug susceptibility pattern of Mycobacterium tuberculosis complex in Zhejiang, China. Biomed Res Int. 2016;2016:8937539. doi:10.1155/2016/8937539

8. Li D, Song Y, Yang P, Li X, Zhang AM, Xia X. Genetic diversity and drug resistance of Mycobacterium tuberculosis in Yunnan, China. $J$ Clin Lab Anal. 2019;33(5):e22884. doi:10.1002/jcla.22884

9. Dou HY, Tseng FC, Lin CW, et al. Molecular epidemiology and evolutionary genetics of Mycobacterium tuberculosis in Taipei. BMC Infect Dis. 2008;8(1):170. doi:10.1186/1471-2334-8-170

10. Pang Y, Zhou Y, Zhao B, et al. Spoligotyping and drug resistance analysis of Mycobacterium tuberculosis strains from national survey in China. PLoS One. 2012;7(3):e32976. doi:10.1371/journal. pone. 0032976
11. Kruuner A, Hoffner SE, Sillastu H, et al. Spread of drug-resistant pulmonary tuberculosis in Estonia. J Clin Microbiol. 2001;39 (9):3339-3345. doi:10.1128/JCM.39.9.3339-3345.2001

12. Anh DD, Borgdorff MW, Van LN, et al. Mycobacterium tuberculosis Beijing genotype emerging in Vietnam. Emerg Infect Dis. 2000;6 (3):302-305. doi:10.3201/eid0603.000312

13. Glynn JR, Jennifer Whiteley PJB, Kremer K, van Soolingen D. Worldwide occurrence of Beijing/W strains of Mycobacterium tuberculosis: a systematic review. Emerg Infect Dis. 2002;8(8):843-849. doi:10.3201/eid0805.020002

14. Stavrum R, Mphahlele M, Ovreas K, et al. High diversity of Mycobacterium tuberculosis genotypes in South Africa and preponderance of mixed infections among ST53 isolates. J Clin Microbiol. 2009;47(6):1848-1856. doi:10.1128/JCM.02167-08

15. Chen L, Pang Y, Ma L, et al. First insight into the molecular epidemiology of Mycobacterium Southwestern China. Biomed Res Int. 2017;2017:2505172.

16. Jagielski T, Augustynowicz-Kopec E, Zozio T, Rastogi N, Zwolska Z. Spoligotype-based comparative population structure analysis of multidrug-resistant and isoniazid-monoresistant Mycobacterium tuberculosis complex clinical isolates in Poland. $J \quad$ Clin Microbiol. 2010;48(11):3899-3909. doi:10.1128/ JCM.00572-10

17. Supply P, Allix C, Lesjean S, et al. Proposal for standardization of optimized mycobacterial interspersed repetitive unit-variable-number tandem repeat typing of Mycobacterium tuberculosis. $J$ Clin Microbiol. 2006;44(12):4498-4510. doi:10.1128/JCM.01392-06

18. Huang TS, Lee SS, Tu HZ, et al. Correlation between pyrazinamide activity and pncA mutations in Mycobacterium tuberculosis isolates in Taiwan. Antimicrob Agents Chemother. 2003;47(11):3672-3673. doi:10.1128/AAC.47.11.3672-3673.2003

19. Cui Z, Wang J, Lu J, Huang X, Zheng R, Hu Z. Evaluation of methods for testing the susceptibility of clinical Mycobacterium tuberculosis isolates to pyrazinamide. J Clin Microbiol. 2013;51 (5):1374-1380. doi:10.1128/JCM.03197-12
Infection and Drug Resistance

\section{Publish your work in this journal}

Infection and Drug Resistance is an international, peer-reviewed openaccess journal that focuses on the optimal treatment of infection (bacterial, fungal and viral) and the development and institution of preventive strategies to minimize the development and spread of resistance. The journal is specifically concerned with the epidemiology of antibiotic resistance and the mechanisms of resistance development and diffusion in both hospitals and the community. The manuscript management system is completely online and includes a very quick and fair peerreview system, which is all easy to use. Visit http://www.dovepress.com/ testimonials.php to read real quotes from published authors. 